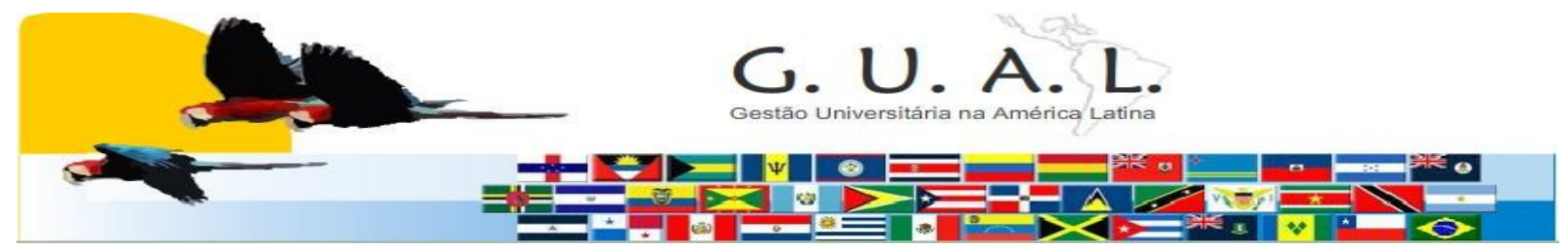

ISSN 1983-4535

\title{
EDUCACIÓN SUPERIOR PÚBLICA EN AMÉRICA LATINA: CARACTERÍSTICAS Y DESAFÍOS
}

\section{PUBLIC HIGHER EDUCATION IN LATIN AMERICA: CHARACTERISTICS AND CHALLENGES}

Guiselle María Garbanzo Vargas, Doutora

Universidad de Costa Rica gmgarban@gmail.com

Recebido em 10/novembro/2011

Aprovado em 13/abril/2012

Sistema de Avaliação: Double Blind Review

Esta obra está sob uma Licença Creative Commons Atribuição-Uso. 


\title{
RESÚMEN
}

La autora realiza una caracterización sobre los principales retos y desafíos que enfrenta la educación superior pública, en especial desde la década de los 90, en el contexto de América Latina. Se parte del papel que ha desempeñado la educación superior pública como promotora de la democracia, estratégica en la modernización política, la legitimidad y estabilidad política, así como su rol fundamental en la movilidad social y en la trasmisión de la cultura. Se hace referencia a las exigencias que la desafiante sociedad actual espera de las instituciones de educación superior pública, ante las cuales se espera una capacidad de respuesta en forma competitiva según la desafiante y emergente sociedad del conocimiento, asegurando así su desarrollo y sostenibilidad social, eso sí en busca de un proyecto educativo y social más inclusivo.

Palabras-clave: Educación superior. América Latina. Desafíos. Globalización.

\begin{abstract}
The author makes a characterization of the principal challenges that public higher education faces, especially since the past 90 decade, in the Latin American context. It begins with the role that public higher education has accomplish to promote the democracy, public modernization strategy, the legitimacy and political stability, and its essential role as an agent of social mobility in the transmission of culture. It refers to the demands of the actual challenges of society that waits to the higher public education, and the capacity to respond these demands in a competitive way in correspondence to the challenges of the emergent society of knowledge. In search of a sustained social development, with a educational and social inclusive project.
\end{abstract}

Keywords: Higher education. Latin America. Challenges. Globalization. 


\section{ANTECEDENTES: CONTEXTO DE LAS UNIVERSIDADES PÚBLICAS LATINOAMERICANAS}

La educación superior en América Latina muestra una vocación de reforma a partir de la década de 1990. Los procesos de acreditación universitaria, cuyo fin es legitimar la oferta ante la demanda han ganado relevancia en la región desde entonces. También, se registran reformas en el financiamiento de las universidades públicas que afectan directamente las funciones de la universidad, en particular respecto de la venta de servicios de docencia e investigación. Por ejemplo, en Argentina se autorizó a las universidades públicas a cobrar aranceles y a tomar sus propias decisiones en materia de admisión, aún cuando se mantiene el compromiso de la educación costeada por el Estado. En México las universidades, en su mayoría, cobran derechos de matrícula bastante altos.

En el nuevo contexto mundial, la tesis que prevalece a partir de la década de 1990 es que la educación superior estatal no se desarrollará como debe, si depende exclusivamente de los fondos públicos, razón por la cual se han impulsado políticas y acciones tendientes a la venta de servicios para adquirir recursos propios, no sin problemas y contradicciones respecto de su función. Esta realidad es particularmente importante en América Latina, ya que la educación superior pública históricamente ha desempeñado un papel vital en la promoción de la democracia. Sus intelectuales han enriquecido el pensamiento nacional. Muchas veces las universidades han servido como vínculos decisivos en la modernización política, así como en la promoción que han realizado de la legitimidad y estabilidad política. Ha cumplido, en buena medida, un papel motor en la movilidad social y desempeña un papel fundamental en la trasmisión de la cultura nacional y en el desarrollo de la identidad nacional.

Según datos del Banco Interamericano de Desarrollo (BID) (1996), la educación superior en América Latina y el Caribe es una actividad prioritaria y no marginal, debido a la propia modernización e integración de la región en una economía globalizada, que exige un cuadro de profesionales altamente competente. Por ello, desempeña un papel decisivo, porque es condición para el progreso económico. Este desarrollo requiere de una reorientación y de recursos, que el BID está anuente a promover y, de hecho, fue el máximo donante externo de recursos en las décadas de 1960 y 1970. Solo entre 1962, que fue el año de su primera inversión, y 1984 desembolsó para estos propósitos $\$ 540.732 .000$. A criterio de personeros del BID, (1996) las universidades poseen cuatro funciones básicas en la región: liderazgo académico, formación para las profesiones, formación técnica y perfeccionamiento, y educación superior general. Por ello, requieren de un adecuado diagnóstico y abordaje. 
La rendición de cuentas que surgió con el desmantelamiento del Estado de Bienestar en la década de 1980, puso en evidencia que las universidades tenían serios problemas de eficiencia y eficacia, entre ellos: un bajo número de estudiantes por profesor, una mayor proporción de personal administrativo respecto del docente, grandes cantidades de alumnos que ingresan pero no llegan a graduarse o con una larga permanencia hasta graduarse. Además, los costos del personal docente y administrativo son desproporcionados en comparación con la inversión de infraestructura.

Otra deficiencia que se le atribuye a la educación superior pública en América Latina es que ofrece programas de estudio desactualizados, poco material didáctico, y estudiantes que pasan por el sistema con un bajo rendimiento académico. Esta situación se presenta tanto en la educación superior pública, como en la privada. También, en algunos casos, el ingreso a la universidad es poco rigoroso y politizado, especialmente en el sector público.

El incremento sostenido de la demanda por educación superior es otro factor que incide en las universidades públicas de la región. La imposibilidad de satisfacer la demanda abre espacios que llenan y continúan satisfaciendo universidades privadas, con la agravante de que la mayoría de ellas ofrece una formación profesional débil.

\section{EDUCACIÓN SUPERIOR PÚBLICA EN EL MARCO DE LA GLOBALIZACIÓN: FUNCIONES E IMPORTANCIA}

La función histórica de la educación superior es transmitir el saber y los conocimientos prácticos que capacitan a los jóvenes para que se incorporen al mundo laboral, y este rol aún goza de validez. Sin embargo, la educación superior se enfrenta hoy a situaciones como la masificación y la necesidad de diversificarla. En los últimos años se discute en particular, sobre el acceso a la educación superior, su calidad, pertinencia e internacionalización (UNESCO, 1999).

La educación superior pública es un recurso nacional e internacional que produce individuos con cultura y formación, cuya profesionalización contribuye a un mejor diálogo y entendimiento entre los pueblos. Los conocimientos que oferta son esenciales en el actual contexto globalizado, altamente complejo y dependiente de la ciencia y la tecnología.

La educación superior es el lugar donde se enseña y forma a las personas que constituyen el capital humano calificado de una nación. Este aporte se considera una de las misiones clásicas de la universidad, sin embargo, en el actual contexto globalizado no 
necesariamente sus esfuerzos se canalizan de ese modo, debido a la masificación y no todos los títulos universitarios aseguran una incorporación eficaz al mundo laboral.

Por otra parte, el civismo sigue siendo fundamental en la educación superior, ya que no se puede reducir la formación a la profesionalización, ya que sus objetivos y perspectivas van más allá de ella. Sigue siendo igualmente válido su carácter de formar espíritus cultos. La nueva sociedad dependerá de las distintas actitudes ante la diferencia y la comprensión de la diversidad (UNESCO, 1999).

La educación superior posee un vínculo fundamental con las políticas económicas orientadas al mundo del trabajo. La globalización ha enmarcado un notable interés con la incorporación laboral de los graduados, para analizar el empleo y desempleo de sus profesionales.

La educación superior, en el marco de la globalización, a criterio de la UNESCO (1999), se enfrenta con una evolución general hacia la liberalización de las economías y la reducción de las responsabilidades gubernamentales en materia de política social, así con los nuevos esquemas de distribución de la riqueza que tendrán repercusiones por lo menos en los primeros años del presente siglo y la evolución de las actitudes sociales con respecto a la riqueza como bien público o privado.

En América Latina y el Caribe se espera que la educación superior sea conducida de manera más eficaz, en relación con el incremento continuo de la inversión en capital humano, efectuada en los sistemas educativos privados y públicos, en vista de que está aumentando el papel desempeñado por esta región en la economía mundial. La educación es un medio, herramienta y estrategia esencial para ayudar a los pueblos a prepararse ante los embates de la globalización y para enfrentar sus posibles repercusiones (Rivero, 1999). Se puede afirmar que en el mundo contemporáneo la educación superior:

- Es un instrumento potenciador de cambios económicos, sociales y culturales indispensables para asumir exitosamente procesos de interdependencia que caracterizarán al siglo XXI.

- El mayor acceso a las oportunidades educativas por parte de individuos y colectividades contribuirá a una mejor comprensión del mundo propio y de los demás, a un mejor acceso al conocimiento y al desarrollo de actividades, competencias y destrezas, que los actuales individuos y grupos sociales requerirán para convivir en armonía, aprender a ser y desarrollarse en un mundo cada vez más complejo. 
Ante el fenómeno de la Globalización la educación debe reorientarse en diferentes direcciones en respuesta a las demandas educativas del mundo globalizado (Beck, 1998), máxime si se considera que:

- Partiendo de que el trabajo ha de sustituirse por conocimiento y capital, entonces una consecuencia política podría ser que el trabajo ha de ser valorado o configurado por el conocimiento. Esto significa que hay que invertir en formación e investigación.

- Los políticos no han comprendido que los verdaderos activos tecnológicos de un país para resolver los complejos problemas del futuro se encuentran en los ciudadanos. Sus conocimientos, sus habilidades, su aportación a la economía mundial (y no la tecnología o el capital) determinan el bienestar de un país.

- Los políticos deben invertir en el conocimiento y la formación, con el propósito de facilitar a los ciudadanos las capacidades y orientaciones para situarse adecuadamente en el escenario y contradicciones de la sociedad mundial.

- Una importante respuesta a la globalización es construir y reconstruir la sociedad del saber y de la cultura. Este planteamiento se concibe bajo una formación continuada, no solo flexible.

- La formación debe situarse en contextos de competencia social, capacidad de dirección, habilidad ante conflictos, comprensión cultural, mentalidad de relación y acceso a las inseguridades y paradojas de la segunda modernidad. El sentido del aprendizaje varía en el nexo transcultural. Se sitúa en la dialéctica de la globalización de la sociedad vivificada.

- Se habla de una transnacionalización de los procesos formativos (universitarios), y de que son necesarios los currículos (estudios de carácter global), siendo esto una herramienta para comprender y dominar las dificultades de la comunicación transcultural y de los conflictos para los estudiantes.

- La transnacionalización de los procesos formativos facilitarían a los estudiantes un mapa cognitivo para comprender la multidimensionalidad y evitar los fallos en la vida y actos "globales".

- La globalización también puede generar conductas reflexivas ante la actual realidad, mediante una aceptación crítica.

De los planteamientos de Beck (1998) se derivan los siguientes elementos en contra del fenómeno de la globalización, desde la perspectiva de la educación:

- La creciente individualización y sus paradojas, que favorece un aprendizaje de conducción de la vida a partir del sí mismo.

- La incapacidad política de enriquecer las oportunidades de formación de la ciudadanía.

- La transnacionalización de los procesos formativos necesarios para comprender y dominar las dificultades de la comunicación transcultural, no se han desarrollado paralelamente en la universidad con los procesos de internacionalización. 
En la actualidad se reconocen como elementos que atentan contra la educación superior pública en el marco de la globalización los siguientes (UNESCO, 2002; Rivero, 1998):

- El actual contexto ha favorecido un aumento en la exclusión, un ensanchamiento de la brecha social, el desempleo y las migraciones, situación que genera desigualdades y desfavorece la educación inclusiva y respetuosa de la diversidad.

- La UNESCO (2001) propone un cambio sustantivo de la educación para atender las demandas del desarrollo humano en el siglo XXI, buscando el cumplimiento de los derechos humanos de todos, hombres y mujeres.

- La cultura está fuertemente influida por los efectos de las tecnologías de la información y la comunicación.

- La economía globalizada impregna toda la vida social, cultural, educativa y comunicacional, que deja a los países en vías de desarrollo en desventaja y sin capacidad de respuesta.

- La globalización provoca una fragmentación social, que hace que millones de personas queden fuera de la sociedad y sus beneficios.

- La globalización puede favorecer en la población con igual fuerza, una actitud activa o pasiva, de aceptación o de rechazo, que hace vulnerable la estabilidad social.

- La globalización implica la imposición de pautas culturales homogéneas a escala planetaria, producto de la revolución de las comunicaciones, el endiosamiento del consumo de los productos impuestos mundialmente.

- El desmoronamiento de las fronteras y el influjo homogenizador llevan a la pérdida de una identidad nacional de valores culturales y sociales.

- Con la entrada masiva de empresas y productos transnacionales, se desborda la demanda laboral, quedando millones de personas desempleadas.

\subsection{Calidad, equidad y pertinencia en la educación superior como desafíos en América Latina}

El debate sobre calidad y pertinencia de la educación superior conduce al enfrentamiento entre la sociedad civil, la educación superior y el Estado. Al respecto, surgen inquietudes sobre cómo establecer la pertinencia social y la función de la educación superior en las estrategias de desarrollo nacional, así como cuánto deben aportar el Estado y el sector privado. Inevitablemente, el nuevo orden socioeconómico impulsa un replanteamiento de las relaciones de fuerza entre esos sectores.

Es un hecho que cada país establece sus propias políticas estratégicas para articular la educación superior en sus planes de desarrollo, debido a que cada uno de ellos tiene sus propias demandas y características. Al respecto, hay acuerdo de que cuando se restringe el aporte estatal a la educación superior se requiere de políticas públicas para subsanar la 
desigualdad de oportunidades. En los países en desarrollo se han implementado políticas de democratización del acceso a la educación superior, como un medio para compensar a los sectores sociales desfavorecidos. Por ello, la igualdad en el acceso tiene importancia significativa en esos países.

En América Latina, a pesar de que existe consenso en que el Estado debe seguir apoyando la educación superior, también hay coincidencia en que se deben encontrar en estas instituciones otras fuentes de financiamiento, por lo que las políticas públicas restringen los recursos que se destinan a ese propósito.

Si bien la reducción de fondos en la educación superior se ha dado tanto en países desarrollados como en vías de desarrollo, ello ha traído consecuencias sociales negativas en América Latina, que agudizan los problemas de la masificación de la educación superior y la consecuente pérdida de calidad y pertinencia (Ruiz, 2001). Esa realidad se refleja en la tendencia en la región a exigir mayor calidad a la educación superior, pero con menos recursos estatales disponibles. En las negociaciones por financiamiento entre el sector universitario y los gobiernos hay múltiples posibilidades. Por ejemplo, países como Brasil, Cuba, Chile y México han llegado a negociar fondos estatales con base en la evaluación. Otros como Cuba, Chile, México y Bolivia con base en sus resultados (Ruiz, 2001).

En un estudio que se llevó a cabo en la década de 1970 sobre la educación superior en América Latina y el Caribe, que incluyó a México, Venezuela, Colombia, Ecuador, Perú, Brasil, Bolivia y Jamaica, se encontró que en todos ellos el crecimiento de la población repercutió en el cambio de la demanda por formación profesional, que requirió democratizar la educación superior (Castrejón, 1979). Esto fue consecuencia de la ampliación demográfica en los niveles primarios educativos, que tuvo como resultado el incremento en la demanda de la educación superior. Ante este problema, los países han respondido en forma variada. Brasil, Jamaica, Perú y Bolivia asumieron una política de "grupos numerosos", tratando de preservar la calidad educativa.

En México, Venezuela, Colombia y Ecuador hubo otras respuestas. En la década de 1970 México amplió las oportunidades de acceso en igualdad de condiciones a la educación superior para evitar presiones políticas de los jóvenes. En Venezuela sucedió lo mismo. Colombia, por su parte, a pesar de que multiplicó sus instituciones, no tuvo la capacidad de ofrecer acceso a toda la demanda estudiantil. Ecuador mantuvo una política de libre acceso.

El acceso a la educación superior es atendido, suficientemente, en cuanto a números, en Brasil, Venezuela y Ecuador. Jamaica, Perú y Bolivia muestran una política de "grupos 
numerosos" y, Colombia y México, a pesar de no evidenciar una política de "grupos numerosos", comparativamente es menor la cantidad de estudiantes que acceden a la educación superior.

La constante expansión del sistema de educación superior pública se ha manifestado en la necesidad de más recursos humanos, físicos y financieros para ofrecer acceso a la educación no excluyente. Si bien llegan más estudiantes de primer ingreso, por otro parte, se registra un problema mayor de empleo de los graduados. Esta situación ha obligado a los distintos países a asumir políticas respecto a la expansión educativa superior.

En cuanto al financiamiento, en este mismo contexto, en la mayoría de los casos se le asigna a la educación superior un porcentaje alto en los gastos totales en educación. En Bolivia se gasta el 33,3\% para atender a 35.000 estudiantes de educación superior. En Brasil el financiamiento de la educación superior recae en el Gobierno federal, que invierte el $42 \%$ del presupuesto. En Colombia el ICFES, distribuye sus fondos en el sistema tecnológico y la educación superior. En Ecuador se toma el 10\% del impuesto sobre la renta para las universidades públicas. En México el costo de la educación superior pública viene de tres partes: el gobierno federal paga el 83,26\%, los gobiernos de los estados el 11,44\% y el 4.96\% recursos propios. En Perú, el principal aporte viene del Gobierno, lo que permite que los estudiantes paguen cuotas bajas y pueda acceder en mayor cantidad estudiantes de bajos recursos económicos. En Venezuela también es costeada en alta proporción por el Estado, y es la que recibe la más alta proporción del presupuesto de los países mencionados.

El desempleo de los graduados es visto no como un problema educativo, sino social, que se mantendrá en la medida en que el desarrollo social de los países de la región y su economía no crezcan en forma paralela. Por ejemplo, Colombia ha multiplicado su oferta, pero es un país con poco desarrollo económico, que limita las posibilidades laborales de los graduados. México tiene como política atender toda la demanda social, con una educación superior incluyente, que ha traído consigo problemas serios de calidad académica y desempleo, precisamente por la falta de desarrollo social y de la poca evolución de la educación superior.

Perú y Bolivia han transformado su sistema de educación superior adoptando una política restrictiva de acceso a las aulas universitarias, permitiéndoles mantener un equilibrio gradual. Estos países presentan un crecimiento limitado, obstaculizando así el crecimiento laboral. Esta situación social ha limitado el acceso a la educación superior a sus jóvenes, en especial a la población económicamente más vulnerable. 
A pesar de que en los países en estudio los gobiernos asumen, en su mayor parte, el problema con sus propios recursos se ha dado una fuerte tendencia a la privatización de los sistemas de educación superior. En Venezuela dieciséis instituciones son privadas y treinta y nueve públicas. En Colombia sesenta y dos universidades de ciento cinco son privadas. En Brasil, seiscientos treinta y ocho de ochocientos cuarenta y ocho son privadas.

La educación superior pública en los cinco países andinos y en México, presenta un deterioro académico, que ha fortalecido el desarrollo y explosión cuantitativa de las universidades privadas, situación que ha influido en la pérdida de imagen de la educación superior pública ante la sociedad. Hace una década las luchas por presupuesto de las universidades públicas eran apoyadas por la mayoría; sin embargo, en la actualidad la opinión pública se ha vuelto indiferente.

Además de los problemas ya mencionados, también se pone de manifiesto en la educación superior en América Latina en la década de 1970, la inestabilidad derivada tanto de cuestiones políticas como de problemas sindicales. Incluso en Colombia algunas universidades públicas han sido cerradas por estos mismos problemas.

En América Latina no se ha logrado aún un equilibrio entre los graduados y el mercado laboral (Castrejón, 1979). Ello muestra que el acceso a la educación superior en igualdad de condiciones debe ir acompañado de un proyecto de desarrollo económico, que reduzca las brechas de desempleo en la región.

\section{CONSIDERACIONES FINALES}

La década de los 90, es para la educación superior en América Latina un período de sustanciales e importantes reformas entorno a su papel ante la sociedad a la cual se debe, ejemplo de ello son los procesos de acreditación, como garantes de su legitimidad. Estas reformas han conducido a las universidades públicas en la región a repensar su rol en un contexto marcado por las exigencias de una naciente sociedad globalizada. En este período también la educación superior pública se ve inmersa en una serie de reformas financieras en cuanto a su sostenimiento estatal, ejemplo de ello son las políticas y acciones relacionadas con la venta de servicios, como medio indispensable para su desarrollo.

La reducción de fondos en la educación superior no es exclusivo de los países en vías de desarrollo, también se ha experimentado en países desarrollados, lo que ha traído consecuencias sociales negativas en América Latina, de manera que agudizan los problemas 
asociados a la masificación de la educación superior y la consecuente limitación de calidad y pertinencia.

La rendición de cuentas a la que se enfrentó la educación superior pública, como consecuencia del desmantelamiento del Estado de Bienestar en la década de 1980, puso en clara evidencia debilidades de este sector educativo, tenían serios problemas de eficiencia y eficacia, por ejemplo relaciones asimétricas entre el número de estudiantes por profesor, una mayor proporción de personal administrativo respecto del docente, cantidades de alumnos que no llegan a graduarse o una larga permanencia estudiantil previo a la graduación, desactualización de sus programas académicos entre otros.

La educación superior, en el marco de la globalización, se encamina hacia la liberalización de las economías y la reducción de las responsabilidades gubernamentales en el campo político y social. En este entorno debe reorientarse respondiendo en forma pertinente a las demandas educativas.

Diferentes elementos atentan contra la educación superior pública en el contexto globalizado, como lo es el favorecer el aumento en la exclusión, el ensanchamiento de la brecha social, problemas asociados al desempleo y las migraciones; lo que no hace otra cosa que generar desigualdades y aumentar la exclusión educativa. La globalización propicia fragmentación social, que hace que millones de personas se limiten de los beneficios sociales.

América Latina continúa realizando esfuerzos en busca de un mejor equilibrio entre los graduados y el mercado laboral, lo que evidencia que el acceso a la educación superior en igualdad de condiciones debe ir acompañado de un proyecto de desarrollo económico, que reduzca las brechas de desempleo en la región, es decir con responsabilidad social.

Finalmente, la educación superior pública en América Latina además, de los retos propios de este sector educativo en el actual contexto globalizado complejo por sí mismo, tiene un doble desafío por ser América Latina la región más desigual de la Orbe, asimetría que no parece reducirse en la actualidad. En este contexto la educación superior pública debe continuar posicionándose con mayor pertinencia social, de manera que mediante la producción de su conocimiento pueda articular mejores proyectos sociales para el contexto al cual se debe como institución pública, a su vez, merece reflexionar de manera propositiva sobre las condiciones reales de acceso y permanencia para las poblaciones marginadas de este espacio educativo, con el propósito de lograr una educación y sociedad más inclusiva para todos. 


\section{BIBLIOGRAFÍA}

BECK, U. (1999). ¿Qué es la globalización? Falacias del globalismo, respuestas a la globalización. Barcelona: Paidos.

CASTREJÓN, D. J. (1979). Educación y Realidad Socioeconómica. Centro de Estudios Educativos. México: A.C.

RUIZ, A. (2001) a. El siglo XXI y el papel de la Universidad: una radiografía de nuestra época y las tendencias en la educación superior. San José, Costa Rica: Editorial de la Universidad de Costa Rica. CONARE.

RUIZ, A. (2001) b. El destino de Costa Rica y la educación superior: el escenario histórico del país, la educación y el papel de la Universidad. San José, Costa Rica: Editorial de la Universidad de Costa Rica. CONARE.

BANCO INETERAMERICANO DE DESARROLLO (BID). (1996). A la búsqueda del siglo XXI: Nuevos caminos de desarrollo en Costa Rica: BID.

\section{FUENTE ELECTRÓNICAS}

UNESCO (1999). La Educación Superior para una nueva sociedad. La visión estudiantil. París, Francia.[Documento WWW]. Recuperado en http.www//unesco.org.syl.34.

UNESCO. (2002). Proyecto Regional de Educación para América Latina y El Caribe. 3-9-

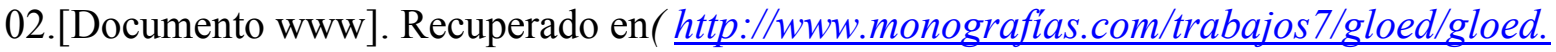
shtml

Htp.www.UNESCO.org. (2002). Proyecto Regional de Educación para América Latina y El Caribe. 3-9-02.[Documento WWW]. Recuperado en:(http://www.monografías.com/ trabajos $7 /$ gloed/gloed.shtml

Htp.www.UNESCO.org. (2002). Proyecto Regional de Educación para América Latina y El Caribe. 3-9-02. (http://www.monografías.com/trabajos7/gloed/gloed.shtml

Htp.www.UNESCO.org. (2002). Proyecto Regional de Educación para América Latina y El Caribe. 3-9-02.

(http://www.monografias.com/trabajos $7 /$ gloed/gloed.shtml

Rivero, H. J. (1999). "Equidad en la educación.” En: Revista Iberoamericana de Educación. 23. Madrid1-26. http://www.campus.oei.org/revista/rie23a03.htm. 